\title{
A Lower Bound on the Sinc Function and Its Application
}

\author{
Yue $\mathrm{Hu}^{1}$ and Cristinel Mortici ${ }^{2,3}$ \\ ${ }^{1}$ School of Mathematics and Information Science, Henan Polytechnic University, Jiaozuo, Henan 454000, China \\ ${ }^{2}$ Valahia University of Târgovişte, 18 Unirii Boulevard, 130082 Târgovişte, Romania \\ ${ }^{3}$ Academy of Romanian Scientists, Splaiul Independenţei 54, 050094 Bucharest, Romania \\ Correspondence should be addressed to Yue Hu; huu3y2@163.com
}

Received 12 March 2014; Revised 24 June 2014; Accepted 26 June 2014; Published 8 July 2014

Academic Editor: Shanhe Wu

Copyright (c) 2014 Y. Hu and C. Mortici. This is an open access article distributed under the Creative Commons Attribution License, which permits unrestricted use, distribution, and reproduction in any medium, provided the original work is properly cited.

A lower bound on the sinc function is given. Application for the sequence $\left\{b_{n}\right\}_{n=1}^{\infty}$ which related to Carleman inequality is given as well.

\section{Introduction}

The sinc function is defined to be

$$
\operatorname{sinc}(x)= \begin{cases}\frac{\sin (x)}{x} & x \neq 0, \\ 1 & x=0 .\end{cases}
$$

This function plays a key role in many areas of mathematics and its applications [1-6].

The following result that provides a lower bound for the sinc is well known as Jordan inequality [7]:

$$
\operatorname{sinc}(x) \geqslant \frac{2}{\pi}, \quad x \in\left[0, \frac{\pi}{2}\right],
$$

where equality holds if and only if $x=\pi / 2$.

This inequality has been further refined by many authors in the past few years [8-35].

In [36], it was proposed that

$$
\operatorname{sinc}(x) \geqslant \frac{\pi^{2}-x^{2}}{\pi^{2}+x^{2}}, \quad x \neq 0 .
$$

We noticed that the lower bound in (3) is the fractional function. Similar result has been reported as follows [1]:

$$
\operatorname{sinc}(x) \geqslant \frac{53}{53+9 x^{2}}, \quad 0 \leqslant x \leqslant \frac{1}{3} .
$$

To the best of the authors' knowledge, few results have been obtained on fractional lower bound for the sinc function. It is the first aim of the present paper to establish the following fractional lower bound for the sinc function.
Theorem 1. For any $x \in[0, \pi]$, one has

$$
\operatorname{sinc}(x) \geqslant \frac{16 \pi^{4}}{\left(3 \pi^{2}+x^{2}\right)^{2}}-1 .
$$

In [37], Yang proved that for any positive integer $m$, the following Carleman type inequality holds:

$$
\sum_{n=1}^{\infty}\left(a_{1} a_{2} \cdots a_{n}\right)^{1 / n}<e \sum_{n=1}^{\infty}\left(1-\sum_{k=1}^{m} \frac{b_{k}}{(n+1)^{k}}\right) a_{n},
$$

whenever $a_{n} \geqslant 0, n=1,2,3, \ldots$, with $0<\sum_{n=1}^{\infty} a_{n}<\infty$, where

$$
\begin{gathered}
b_{0}=1, \\
b_{n}=\frac{1}{n}\left(\frac{1}{n+1}-\sum_{k=0}^{n-2} \frac{b_{n-1-k}}{k+1}\right), \quad(n=1,2, \ldots) .
\end{gathered}
$$

From a mathematical point of view, the sequence $\left\{b_{n}\right\}_{n=1}^{\infty}$ has very interesting properties. Yang [38] and Gyllenberg and Ping [39] have proved that, for any positive integer $n$,

$$
\begin{gathered}
b_{n}>0, \\
b_{n}<\frac{1}{n(n+1)} .
\end{gathered}
$$


In [40], the authors proved that

$$
\begin{gathered}
\lim _{n \rightarrow \infty} \frac{b_{n+1}}{b_{n}}=1, \\
e b_{n}=\int_{0}^{1} x^{n-2} h(x) d x, \quad n \geqslant 2,
\end{gathered}
$$

where

$$
h(x)=x^{x+1}(1-x)^{1-x} \operatorname{sinc}(\pi x) .
$$

As an application of Theorem 1, it is the second aim of the present paper to give a better upper bound on the sequence $\left\{b_{n}\right\}_{n=1}^{\infty}$.

Theorem 2. For any positive integer $n \geqslant 2$, one has

$$
e b_{n}<\frac{1}{n(n+1)}-\frac{2-4 / \pi}{n(n+1)(n+2)} .
$$

\section{The Proof of Theorem 1}

The proof is not based on (3). We first prove the following result.

Lemma 3. For any $x \in(\pi-1 / 3, \pi]$, one has

$$
\operatorname{sinc}(x) \geqslant \frac{16 \pi^{4}}{\left(3 \pi^{2}+x^{2}\right)^{2}}-1 .
$$

Proof. Set $x=\pi-t, 0 \leqslant t<1 / 3$. Then inequality (13) is equivalent to

$$
\pi-t+\sin t \geqslant \frac{16 \pi^{4}(\pi-t)}{\left(3 \pi^{2}+(\pi-t)^{2}\right)^{2}} .
$$

To prove (14) by (4), it is enough to prove that

$$
(\pi-t)+\frac{53 t}{53+9 t^{2}} \geqslant \frac{16 \pi^{4}(\pi-t)}{\left(3 \pi^{2}+(\pi-t)^{2}\right)^{2}}
$$

namely,

$$
\frac{(\pi-t)\left(53+9 t^{2}\right)+53 t}{\left(53+9 t^{2}\right)} \geqslant \frac{16 \pi^{4}(\pi-t)}{\left(3 \pi^{2}+(\pi-t)^{2}\right)^{2}} .
$$

Next we prove (16). Let

$$
\begin{aligned}
g(t)= & (\pi-t)\left(53+9 t^{2}\right)\left(3 \pi^{2}+(\pi-t)^{2}\right)^{2} \\
& +53 t\left(3 \pi^{2}+(\pi-t)^{2}\right)^{2} \\
& -16 \pi^{4}(\pi-t)\left(53+9 t^{2}\right) .
\end{aligned}
$$

We need only to prove that $g(t) \geqslant 0$. Elementary calculations reveal that

$$
\begin{aligned}
g(t)=t^{2}( & -9 t^{5}+45 \pi t^{4}-144 \pi^{2} t^{3}+\left(53 \pi+252 \pi^{3}\right) t^{2} \\
- & \left.4 \pi^{2}\left(36 \pi^{2}+53\right) t+636 \pi^{3}\right) .
\end{aligned}
$$

Noting that, for $0 \leqslant t<1 / 3$, we have

$$
\begin{aligned}
-9 t^{5} & >-\frac{1}{27} \\
-144 \pi^{2} t^{3} & >-\frac{16 \pi^{2}}{3} \\
-4 \pi^{2}\left(36 \pi^{2}+53\right) t & >-\frac{4}{3} \pi^{2}\left(36 \pi^{2}+53\right) .
\end{aligned}
$$

Thus, from (19) and (18), we get

$$
g(t) \geqslant t^{2}\left(636 \pi^{3}-\frac{1}{27}-\frac{16 \pi^{2}}{3}-\frac{4 \pi^{2}\left(36 \pi^{2}+53\right)}{3}\right) \geqslant 0 .
$$

This completes the proof. Now we prove Theorem 1 .

Proof. By using the power series expansions of $\sin (x)$ and $16 \pi^{4} /\left(3 \pi^{2}+x^{2}\right)^{2}$, we find that

$$
\begin{aligned}
1+ & \operatorname{sinc}(x)-\frac{16 \pi^{4}}{\left(3 \pi^{2}+x^{2}\right)^{2}} \\
& =\frac{2}{9}+\sum_{n=1}^{\infty}(-1)^{n-1} u_{n}\left(\frac{x^{2}}{\pi^{2}}\right)^{n},
\end{aligned}
$$

where

$$
u_{n}=\frac{16(n+1)}{3^{n+2}}-\frac{\pi^{2 n}}{(2 n+1) !}
$$

Set $x^{2} / \pi^{2}=t, 0 \leqslant t \leqslant 1$. Consider the function $f(t)$ defined by

$$
f(t)=\frac{2}{9}+\sum_{n=1}^{\infty}(-1)^{n-1} u_{n} t^{n}
$$

From (21), we get $f(0)=2 / 9$ and $f(1)=0$. Lemma 3 implies

$$
f(t) \geqslant 0, \quad t_{0}<t \leqslant 1,
$$

where

$$
t_{0}=\left(1-\frac{1}{3 \pi}\right)^{2} \approx 0.79 \ldots .
$$

Elementary calculations reveal that for $n \geqslant 4$,

$$
\frac{16(2 n+1)}{3^{3}}>\frac{\left(3 \pi^{2}\right)^{n}}{(2 n+1) !} .
$$

Hence, for $n \geqslant 4$, we have

$$
u_{n}>0
$$

$$
u_{n}-u_{n+1}=\frac{16(2 n+1)}{3^{n+3}}-\frac{\pi^{2 n}}{(2 n+1) !}+\frac{\pi^{2 n+2}}{(2 n+3) !}>0
$$


Therefore,

$$
f(t) \geqslant \frac{2}{9}+\sum_{n=1}^{6}(-1)^{n-1} u_{n} t^{n}
$$

If we set

$$
g(t)=\frac{2}{9}+\sum_{n=1}^{6}(-1)^{n-1} u_{n} t^{n}
$$

then we have

$$
g(0)=\frac{2}{9}>0, \quad g(1)<f(1)=0 .
$$

The intermediate value theorem implies that there must be at least one root $c$ with $(0,1)$ such that $g(c)=0$. Using Maple, we find that on the open interval $(0,1)$ the equation $g(t)=0$ has a unique real root $t_{1} \approx 0.89 \cdots$.

Hence, from (28) we get

$$
f(t) \geqslant 0, \quad t \in\left[0, t_{1}\right] .
$$

By (21), (24), and (31), Theorem 1 follows.

\section{The Proof of Theorem 2}

First, we need an auxiliary result.

Lemma 4. For any $x \in[0,1 / 2]$, one has

$$
\operatorname{sinc}^{2}(\pi x) \geqslant \frac{1-2 x+x^{2}}{1-x+x^{2}}
$$

Proof. By letting $x=1 / 2-t / 2 \pi, 0 \leqslant t \leqslant \pi$, the requested inequality can be equivalently written as

$$
\cos t \geqslant \frac{t^{2}}{2}+\frac{8 \pi^{4}}{t^{2}+3 \pi^{2}}-\frac{5 \pi^{2}+2}{2},
$$

so it suffices to show that the function

$$
G(t)=\cos t-\frac{t^{2}}{2}-\frac{8 \pi^{4}}{t^{2}+3 \pi^{2}}+\frac{5 \pi^{2}+2}{2}
$$

is negative on $0 \leqslant t \leqslant \pi$. Theorem 1 implies

$$
G^{\prime}(t)<0 \text {. }
$$

Hence,

$$
G(t) \geqslant G(\pi)=0 .
$$

The required inequality follows. Now we prove Theorem 2 .

Proof. Let

$$
H(x)= \begin{cases}x^{x}(1-x)^{-x} \operatorname{sinc}(\pi x), & 0<x<1 \\ 1 & x=0,1 .\end{cases}
$$

We first consider the case $0 \leqslant x \leqslant 1 / 2$.
Taking the natural log gives

$$
\ln H(x)=(x-1) \ln x-x \ln (1-x)+\ln \sin \pi x-\ln \pi \text {. }
$$

Taking the second derivative of both sides of (38), we have

$$
\frac{H H^{\prime \prime}-H^{\prime 2}}{H^{2}}=\frac{x^{2}-x+1}{x^{2}(1-x)^{2}}-\pi^{2} \csc ^{2}(\pi x) .
$$

By Lemma 4, it follows that

$$
\frac{H H^{\prime \prime}-H^{\prime 2}}{H^{2}}>0 \text {. }
$$

Thus,

$$
H^{\prime \prime}>0 \text {, }
$$

and therefore for $0 \leqslant x \leqslant 1 / 2$, we have

$$
\begin{aligned}
H(x) & \leqslant(1-2 x) H(0)+2 x H\left(\frac{1}{2}\right) \\
& =\left(2-\frac{4}{\pi}\right)(-x)+1 .
\end{aligned}
$$

For the case $1 / 2<x \leqslant 1$, since $H(1 / 2)=2 / \pi, H(1)=1$, and $H$ is concave up, it follows that

$$
\begin{aligned}
H(x) & \leqslant 2(1-x) H\left(\frac{1}{2}\right)+(2 x-1) H(1) \\
& =\left(2-\frac{4}{\pi}\right)(x-1)+1
\end{aligned}
$$

Using (10) from (42) and (43), we have

$$
\begin{aligned}
e b_{n}= & \int_{0}^{1} x^{n-2} h(x) d x=\int_{0}^{1} H(x)\left(x^{n-1}-x^{n}\right) d x \\
\leqslant & \int_{0}^{1 / 2}\left[1-\left(2-\frac{4}{\pi}\right) x\right]\left(x^{n-1}-x^{n}\right) d x \\
& +\int_{1 / 2}^{1}\left[1+\left(2-\frac{4}{\pi}\right)(x-1)\right]\left(x^{n-1}-x^{n}\right) d x \\
= & \frac{1}{n(n+1)}-\left(2-\frac{4}{\pi}\right) \frac{2^{n+2}-n-4}{n(n+1)(n+2) 2^{n+1}} \\
< & \frac{1}{n(n+1)}-\frac{2-4 / \pi}{n(n+1)(n+2)} .
\end{aligned}
$$

This proves Theorem 2 .

\section{Conflict of Interests}

There is no conflict of interests regarding the publication of this paper.

\section{Acknowledgments}

The authors are very grateful to the anonymous referees and the editor for their insightful comments and suggestions. The work of the second author was supported by a Grant of the Romanian National Authority for Scientific Research, CNCSUEFISCDI project no. PN-II-ID-PCE-2011-3-0087. 


\section{References}

[1] J. Kuang, Applied Inequalities, Shandong Science and Technology Press, 3rd edition, 2004.

[2] F. Stenger, Numerical Methods Based on Sinc and Analytic Functions, vol. 20 of Springer Series in Computational Mathematics, Springer, New York, NY, USA, 1993.

[3] J. P. Boyd, Chebyshev and Fourier Spectral Methods, Dover, New York, NY, USA, 2nd edition, 2000.

[4] D. Borwein, J. M. Borwein, and I. E. Leonard, "Lp norms and the sinc function," The American Mathematical Monthly, vol. 117, no. 6, pp. 528-539, 2010.

[5] D. Borwein and J. M. Borwein, "Some remarkable properties of sinc and related integrals," Ramanujan Journal, vol. 5, no. 1, pp. 73-89, 2001.

[6] W. B. Gearhart and H. S. Schultz, "The function $\sin (\mathrm{x}) / \mathrm{x}$," The College Mathematics Journal, vol. 2, no. 2, pp. 90-99, 1990.

[7] D. S. Mitrinovic, Analytic Inequalities, Springer, New York, NY, USA, 1970.

[8] S.-P. Zeng and Y.-S. Wu, "Some new inequalities of Jordan type for sine," The Scientific World Journal, vol. 2013, Article ID 834159, 5 pages, 2013.

[9] R. P. Agarwal, Y. Kim, and S. K. Sen, "A new refined jordan's inequality and its application," Mathematical Inequalities and Applications, vol. 12, no. 2, pp. 255-264, 2009.

[10] Á. Baricz, "Some inequalities involving generalized bessel functions," Mathematical Inequalities and Applications, vol. 10, no. 4, pp. 827-842, 2007.

[11] A. Baricz, "Jordan-type inequalities for generalized Bessel functions," Journal of Inequalities in Pure and Applied Mathematics, vol. 9, no. 2, article 39, 2008.

[12] A. Baricz, "Geometric properties of generalized Bessel functions," Publicationes Mathematicae Debrecen, vol. 73, no. 1-2, pp. 155-178, 2008.

[13] L. Debnath and C. Zhao, "New strengthened Jordan's inequality and its applications," Applied Mathematics Letters, vol. 16, no. 4, pp. 557-560, 2003.

[14] J. Li, "An identity related to Jordan's inequality," International Journal of Mathematics and Mathematical Sciences, vol. 2006, Article ID 76782, 6 pages, 2006.

[15] J. L. Li and Y. L. Li, "On the strengthened Jordan's inequality," Journal of Inequalities and Applications, vol. 2007, Article ID 74328, 9 pages, 2007.

[16] D. Niu, Z. Huo, J. Cao, and F. Qi, "A general refinement of Jordan's inequality and a refinement of L. Yang's inequality," Integral Transforms and Special Functions, vol. 19, no. 3-4, pp. 157-164, 2008.

[17] A. Y. Özban, "A new refined form of Jordan's inequality and its applications," Applied Mathematics Letters, vol. 19, no. 2, pp. 155160, 2006.

[18] F. Qi and Q. D. Hao, "Refinements and sharpenings of Jordan's and Kober's inequality," Mathematical Inequalities \& Applications, vol. 8, no. 3, pp. 116-120, 1998.

[19] F. Qi, L. Cui, and S. Xu, "Some inequalities constructed by Tchebysheff's integral inequality," Mathematical Inequalities and Applications, vol. 2, no. 4, pp. 517-528, 1999.

[20] F. Qi, “Jordan's inequality: refinements, generalizations, applications and related problems," RGMIA Research Report Collection, vol. 9, no. 3, article 12, 2006.

[21] F. Qi, D.-W. Niu, and B.-N. Guo, "Refinements, generalizations, and applications of Jordan's inequality and related problems,"
Journal of Inequalities and Applications, vol. 2009, Article ID 271923, 52 pages, 2009.

[22] J. Sandor, "On the concavity of sinx/x," Octogon Mathematical Magazine, vol. 13, no. 1, pp. 406-407, 2005.

[23] S. H. Wu, "On generalizations and refinements of Jordan type inequality," RGMIA Research Report Collection, vol. 7, article 2, 2004.

[24] S. H. Wu, "On generalizations and refinements of Jordan type inequality," Octogon Mathematical Magazine, vol. 12, no. 1, pp. 267-272, 2004.

[25] S. Wu and L. Debnath, "A new generalized and sharp version of Jordan's inequality and its applications to the improvement of the Yang Le inequality," Applied Mathematics Letters, vol. 19, no. 12, pp. 1378-1384, 2006.

[26] S. Wu and L. Debnath, "A new generalized and sharp version of Jordan's inequality and its applications to the improvement of the Yang Le inequality.II," Applied Mathematics Letters, vol. 20, no. 5, pp. 532-538, 2007.

[27] S. Wu and L. Debnath, "Jordan-type inequalities for differentiable functions and their applications," Applied Mathematics Letters, vol. 21, no. 8, pp. 803-809, 2008.

[28] S. Wu and H. M. Srivastava, "A further refinement of a Jordan type inequality and its application," Applied Mathematics and Computation, vol. 197, no. 2, pp. 914-923, 2008.

[29] S. Wu, H. M. Srivastava, and L. Debnath, "Some refined families of Jordan-type inequalities and their applications," Integral Transforms and Special Functions, vol. 19, no. 3-4, pp. 183-193, 2008.

[30] L. Zhu, "Sharpening Jordan's inequality and Yang Le inequality. II," Applied Mathematics Letters, vol. 19, no. 9, pp. 990-994, 2006.

[31] L. Zhu, "Sharpening of Jordan's inequalities and its applications," Mathematical Inequalities \& Applications, vol. 9, no. 1, pp. 103-106, 2006.

[32] L. Zhu, "A general refinement of Jordan-type inequality," Computers and Mathematics with Applications, vol. 55, no. 11, pp. 2498-2505, 2008.

[33] L. Zhu, "General forms of Jordan and Yang Le inequalities," Applied Mathematics Letters, vol. 22, no. 2, pp. 236-241, 2009.

[34] L. Zhu and J. Sun, "Six new Redheffer-type inequalities for circular and hyperbolic functions," Computers \& Mathematics with Applications, vol. 56, no. 2, pp. 522-529, 2008.

[35] Y. Qiu and L. Zhu, "The best approximation of the sinc function by a polynomial of degree $n$ with the square norm," Journal of Inequalities and Applications, vol. 2010, Article ID 307892, 12 pages, 2010.

[36] R. Redheffer, "Problem 5642," The American Mathematical Monthly, vol. 75, no. 10, pp. 1125-1126, 1968.

[37] X. Yang, "Approximations for constant $e$ and their applications," Journal of Mathematical Analysis and Applications, vol. 262, no. 2, pp. 651-659, 2001.

[38] X. Yang, "On Carleman's inequality," Journal of Mathematical Analysis and Applications, vol. 253, no. 2, pp. 691-694, 2001.

[39] M. Gyllenberg and Y. Ping, "On a conjecture by Yang," Journal of Mathematical Analysis and Applications, vol. 264, no. 2, pp. 687-690, 2001

[40] Y. Hu and C. Mortici, "On the coefficients of an expansion of $(1+1 / x)^{x}$ related to Carleman's inequality," http://arxiv.org/ abs/1401.2236. 


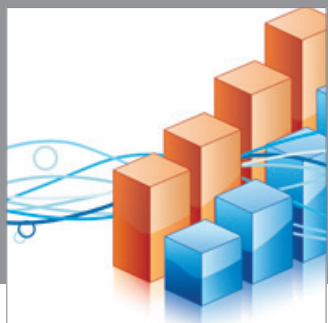

Advances in

Operations Research

mansans

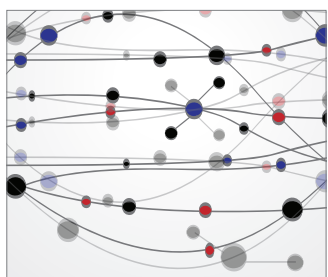

The Scientific World Journal
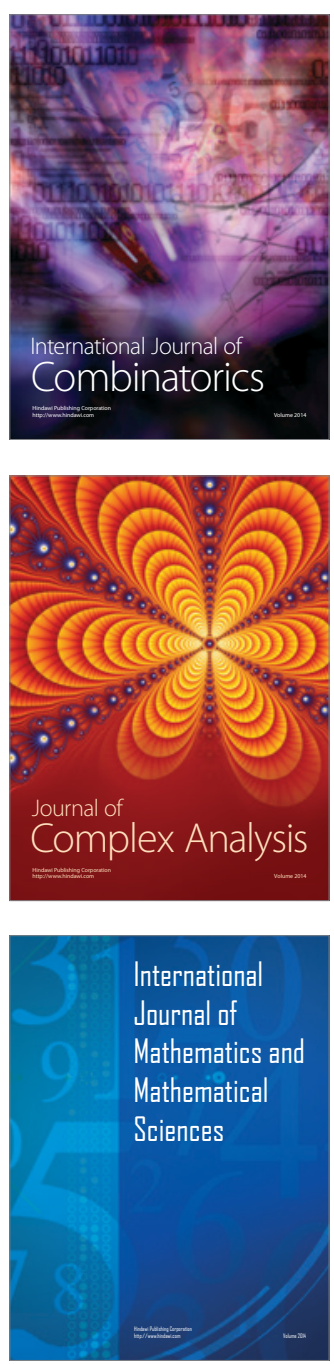
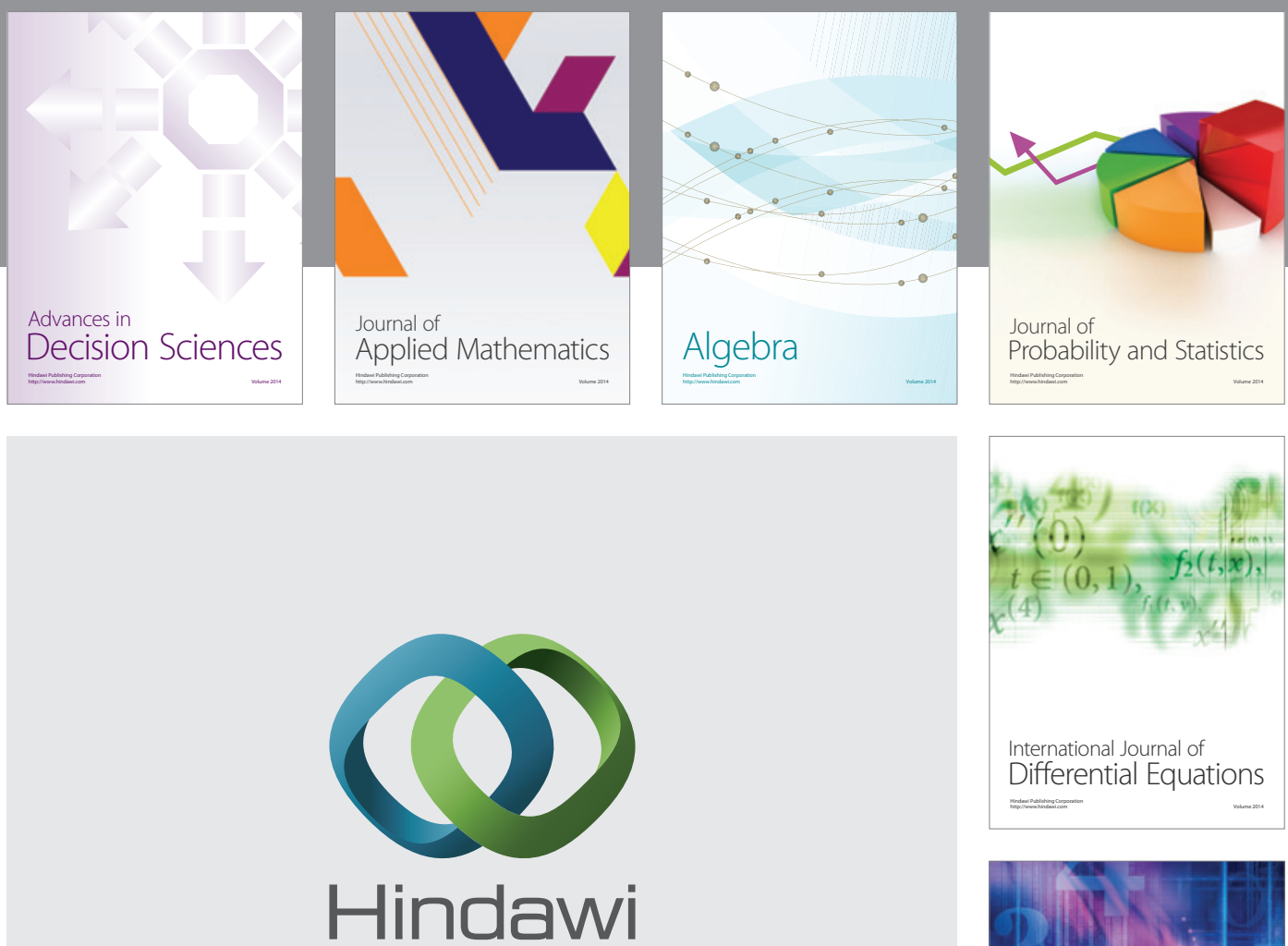

Submit your manuscripts at http://www.hindawi.com
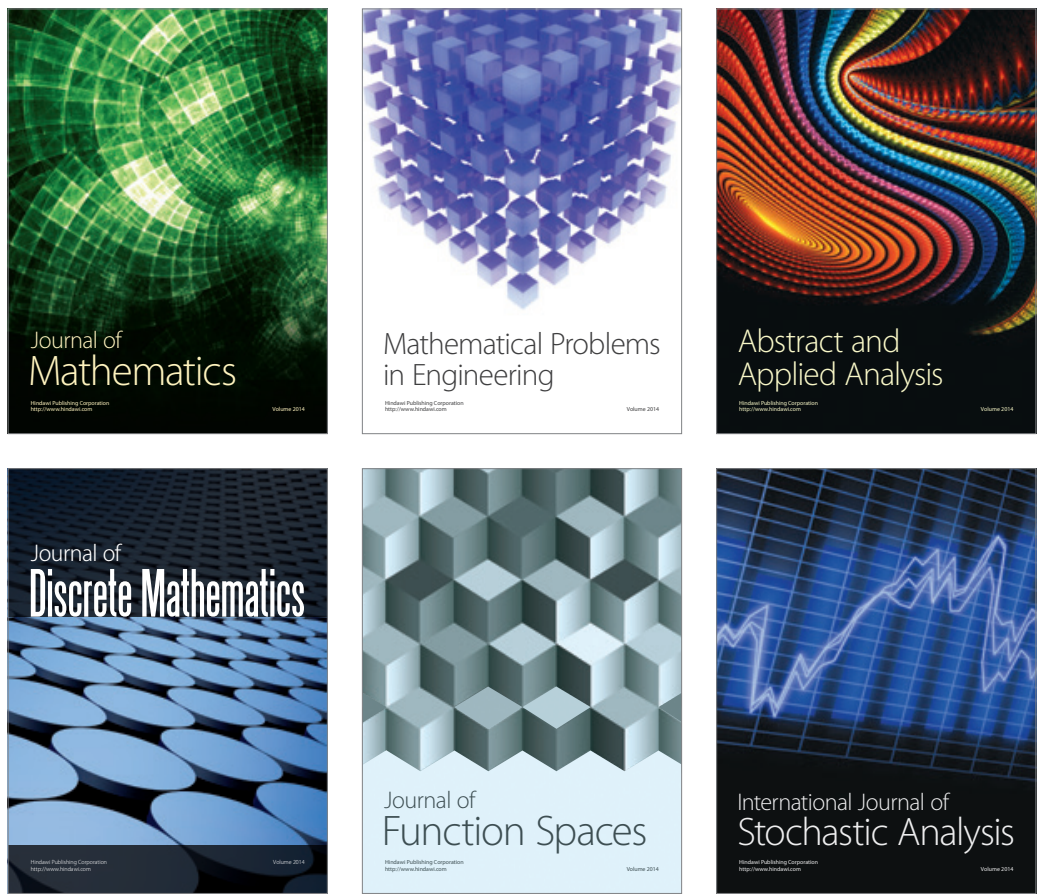

Journal of

Function Spaces

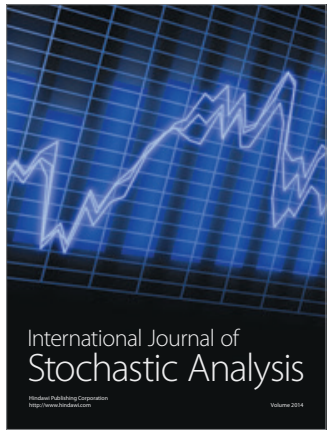

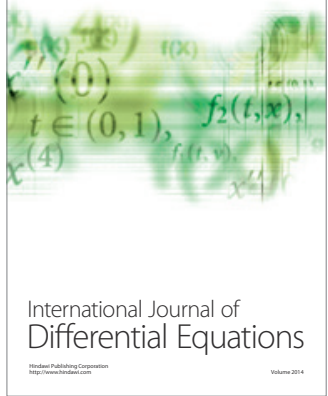
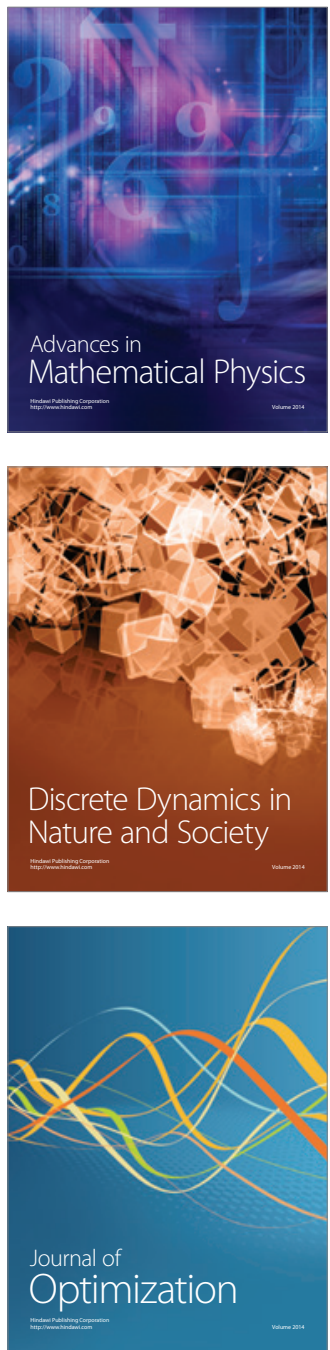